\title{
The Predictive Value of C-reactive Protein to Albumin Ratio to Detect Contrast-induced Acute Kidney Injury in Patients with Acute Myocardial Infarction Undergoing Percutaneous Coronary Intervention
}

\author{
Akut Miyokart Enfarktüsü Nedeniyle Perkütan Koroner Girișim Uygulanan Hastalarda \\ Kontrast Kaynaklı Akut Böbrek Hasarı Tespitinde C-reaktif' Protein - Albümin Oranının \\ Öngördürücü Değeri
}

\author{
Ahmet Karakurt \\ Department of Cardiology, Faculty of Medicine, Kafkas University, Kars, Turkey
}

\begin{abstract}
Aim: The present study aims to investigate the predictive value of the peak $C$-reactive protein to reduced albumin ratio (CAR) in the development of contrast-induced acute kidney injury (Cl-AKI) in patients with acute myocardial infarction (AMI) undergoing primary percutaneous coronary intervention ( $p P C l)$.

Material and Method: Two hundred and ten patients with non$S T$ and ST-elevation AMI undergoing $\mathrm{PPCl}$ were recorded. Renal function parameters and highest CAO values of the patients after $\mathrm{pPCl}$ were recorded from the patient files. The patient population was divided intothe low-, mid-, and high-CAR tertiles (all $n=70$ ) after their $C A R$ values were ranked from least to greatest.

Results: Eighty-nine (42.38\%) patients developed Cl-AKI. The absolute increase in serum creatinine ( $\mathrm{sCr}$ ) was significantly higher in patients in the high-CAR tertile $(0.31$, interquartile range (IQR): 1.925-0.555) than in the low-CAR (0.2, IQR: 0.118$0.27)$ and mid-CAR tertiles (0.2, IQR: 0.13-0.33; $p<0.001)$. The area under the curve of CAR was0.689 (95\% Cl: 0.614-0.763; $p<0.001)$ in the receiver operating characteristic analysis. After adjusting for other risk factors of $\mathrm{Cl}-\mathrm{AKI}, \mathrm{CAR}$ remained predictors of the development of Cl-AKI (OR: 1.345, 95\% Cl: 1.009 1.794, $p=0.043$ ).
\end{abstract}

Conclusion: The elevated CAR value is independent predictors in the development of $\mathrm{Cl}-\mathrm{AKI}$ in patients with $\mathrm{AMI}$ undergoing $\mathrm{pPCl}$. In this patient group, the elevated CAR value after PPKG may alert us to more improve $\mathrm{Cl}-\mathrm{AKI}$.

Key words: acute myocardial infarction; $C$-reactive protein to albumin ratio; contrast-induced acute kidney injury
ÖZET

Amaç: Bu çalıșmada akut miyokart enfarktüsü (AME) nedeniyle primerperkütan koroner girișim ( $P P K G$ ) yapılan hastalarda kontrastkaynaklı akut böbrek hasarı $(K K-A B H)$ gelișiminde C-reaktif protein - albümin oranının (CAO) öngördürücü değerini belirlenmesi amaçlamaktadır.

Materyal ve Metot: Çalıșmaya pPKG uygulanan ST-elevasyonsuz ve ST-elevasyonu 210 AME'li hasta dâhil edildi. Hastaların pPKG sonrası böbrek fonksiyon parametreleri ve en yüksek CAO değerleri hasta dosyalarından kaydedildi. Çalıșma popülasyonunun CAO değerleri en küçükten en büyüğe doğru sıralandıktan sonra düșük-, orta- ve yüksek-CAO olmak üzere üç guruba ayrıldı (her $n=70$ ).

Bulgular: Seksen dokuz hastada $(\% 42,38) K K-A B H$ geliști. Serum kreatininindeki (sCr) mutlak artıșı, yüksek-CAO gurubunda $[0,31$, interquartilerange (IQR): 1,925-0,555], düșük- (0,2, IQR: 0,118$0,27)$ ve orta-CAO'dan önemli ölçüde daha yüksek bulundu $(0,2$, IQR: 0,13-0,33; $p<0,001)$. Receiveroperatingcharacteristic analizde CAO'ya ait eğri altındaki alan 0,689 (95 \% Cl: 0,614-0,763; $p<0,001)$ bulundu. $K K-A B H$ gelișimine neden olacak diğer risk faktörleri ayarlandıktan sonra yapılan çok değișkenli regresyon analizi, CAO'nun KK-ABH gelișimini öngördürecek olduğunu gösterdi (OR: 1,345, \%95 Cl: 1,009-1,794, $p=0,043$ ).

Sonuç: Yüksek CAO değerleri, $p P C l$ uygulanan AME hastalarında $K K-A B H$ gelișiminde bağımsız öngördürücüdür. Bu hasta grubunda, pPKG sonrası yüksek $C A O$ değeri $K K-A B H$ 'nın gelișebileceğ yönünde bizi uyarmalıdır.

Anahtar kelimeler: akut miyokart enfarktüsü; C-reaktif proteinin albümin oranı; kontrast-kaynaklı akut böbrek hasarı 


\section{Introduction}

Contrast induced acute kidney injury (CI-AKI) is the third leading cause of iatrogenic renal insufficiency, which usually occurs within hours following percutaneous coronary procedures ${ }^{1,2}$. Its incidence is around $2 \%$ in the general population, but increases to $50 \%$ in high-risk groups especially after percutaneous coronary intervention $(\mathrm{PCI})^{3}$.

Even though, the mechanism has not been clearly understood, the inflammation plays an important role in the pathophysiologic reason of CI-AKI ${ }^{4}$. C-reactive protein (CRP) is well-known marker of systemic inflammation, and it's shown that the elevated CRP level is associated with cardiovascular adverse events and CI-AKI in patient with acute myocardial infarction (AMI) ${ }^{5}$. Serum albumin (sALB), which is the most abundant protein in human plasma, is an acute phase reactant. It was shown that SALB concentration was reduced during the systemic inflammation and is associated with poor clinical outcomes ${ }^{6,7}$.

In the recent years, the $\mathrm{C}$-reactive protein to albumin ratio (CAR) as a new marker of systemic inflammation has been investigated in various diseases, especially sepsis and malignancies ${ }^{8,9}$. AMI is an inflammatory process and based on the theory that increased inflammation may increase the risk of CI-AKI development, we aimed to evaluate the predictive value of CAR and sALB to predicting the development of CI-AKI in patients with non-ST and ST-elevation AMI.

\section{Material and Method}

\section{Study Population}

We retrospectively screened the patients who underwent primary PCI for AMI between January 2017 and March 2019. The sample sizes for the groups were calculated using the OpenEpi sample size calculation program (https://www. openepi. com/SampleSize/ SSCC. htm). Accordingly, we enrolled 89 and 121 in the CI-AKI group and no-CI-AKI group, respectively (total n: 210). Demographic, clinic, laboratory, echocardiographic and angiographic data obtained from the patient files end hospital digital record. An AMI was diagnosed according to the criteria recommended by the 2017 European Society of Cardiology Guidelines ${ }^{10}$. The study was started after obtaining the written approval from the local ethics board (registration number: 80576354-050-99/176); the research protocols were as per the Declaration of Helsinki.
Inclusion criteria; (1) initial eGFR > $60 \mathrm{ml} / \mathrm{min}$; (2) no history of acute or chronic kidney disease; (3) the initial $s \mathrm{Cr}$ value is within the normal range $(0.7-1.2$ $\mathrm{mg} / \mathrm{dl})$. Patients who were diagnosed with stable or unstable angina pectoris, hypotension (arterial blood pressure below $90 / 60 \mathrm{mmHg}$ during hospital stay), decompensated congestive heart failure or pulmonary edema, use of intra-arterial balloon pump (IABP) therapy, cardiogenic shock, coronary artery bypass grafting operation and/or PCI and prior contrast media exposure within 1 mount were excluded. Also, patients with anemia, a history of myocarditis, pericarditis, severe valve disease, active infection, chronic pulmonary or liver disease, malignancy and connective tissue disorder and use of nephrotoxic drugs previous in 1 week were excluded from the study.

\section{Laboratory Measurements}

Detailed laboratory parameters of patients were obtained at admission and serum creatinine $(\mathrm{sCr})$, CRP and sALB levels were measured at 24, 48, 72 and 96 hours after PCI during the hospital stay. sCr, CRP and sALB levels was measured using Electrochemiluminescent (ECL) processes using Cobas 6000 system (Roche, Minato-ku, Tokyo). The CAR was calculated as the ratio of peak CRP to the lower sALB.

Acute Kidney Injury Network (AKIN) and Risk, Injury, Failure, Loss of kidney function, and End-stage kidney disease (RIFLE) are used frequently in terms of AKI classification ${ }^{11,12}$. However, the definition of the European Society of Urogenital Radiology (ESUR) is widely accepted for the contrast-induced acute kidney injury (CI-AKI) ${ }^{3,13}$. According to this definition, an absolute increase of $\geq 0.5 \mathrm{mg} / \mathrm{dl}$ or $44 \mathrm{mmol} / \mathrm{L}$ or $\geq 25 \%$ in the basal $\mathrm{sCr}$ value within $24-72$ hours after contrast exposure makes the diagnosis of CI-AKI. In the present study, we preferred to use the definition of the ESUR for CI-AKI diagnosis and the groups were constituted according to the aforementioned definition. The estimated glomerular filtration rate (eGFR) was calculated automatically by Cobas 6000 system using the chronic kidney disease epidemiology collaboration equation $\left[\mathrm{eGFR}=141 * \min (\mathrm{sCr} / \kappa, 1) \alpha^{*}\right.$ $\max (\mathrm{sCr} / \kappa, 1)-1.209^{*} 0.993$ Age $^{*} 1.018$ (if female) * 1.159 (if black); $\kappa$ is 0.7 for females and 0.9 for males, $\alpha$ is -0.329 for females and -0.411 for males, min indicates the minimum of $s \mathrm{Cr} / \kappa$ or 1 , and max indicates the maximum of $s \mathrm{Cr} / \kappa$ or 1$]^{14}$. On the day of peak $\mathrm{sCr}$, 
the total fluid intake and volume of urine output were recorded from the daily patient observation forms.

Left ventricular ejection fraction (LVEF) was measured on admission with two-dimensional echocardiography using the modified Simpson's method, using a Vivid 7 cardiovascular ultrasound system (General Electric, Mil-Waukee, Wisconsin) equipped with a 3.6 $\mathrm{MHz}$ transducer.

\section{Coronary Angiography}

Coronary angiography and pPCI was performed by a coronary interventional cardiologist team, according to standard Judkins technique, using the femoral approach and 6-F diagnostic and 6 or 7-F guiding catheters, respectively. Infarct related artery (IRA) was defined as the presence of $\geq 50 \%$ luminal diameter narrowing in culprit major coronary artery which is compatible with presented ST segment change on electrocardiography. Before the pPCI, all patients received $300 \mathrm{mg}$ acetylsalicylic acid, $600 \mathrm{mg}$ clopidogrel and a bolus of $80-100 \mathrm{U} / \mathrm{kg}$ unfractionated heparin, if deemed necessary, followed by an additional bolus heparin and nitroglycerin during the procedure. The stent length was given as the sum of the all implanted stents to the IRA and the other coronary arteries with the critical stenosis in the same patient. The syntax score which is an angiographic grading tool to determine the complexity of the coronary artery disease was calculated using the syntax score calculator. (http:// www. syntaxscore. com/calculator/start. htm). In all patients, iso-osmolar CM was used, however, its volume was left to the discretion of the coronary interventional cardiologist.

\section{Statistical Analysis}

Continuous variables were tested for normal distribution through the Kolmogorov-Smirnov test and homogeneity of variance with the Levene's test. Categorical variables were given as percentages, parametric variables were presented as means \pm SD and non-parametric variables were presented as median (25th-75th). Categorical variables analyses were compared by the Pearson Chi-Square test or the Fisher Exact test, parametric continuous variables analyses were compared by Independent Sample T test and nonparametric continuous variables and categorical variables were compared by the Mann-Whitney $U$ test. The strength of association between CAR, creatinine, albumin and other values were performed using Spearman correlation (for non-parametric variables) or Pearson correlation (for parametric variables). The univariate and multivariable logistic regression analysis was used to evaluate the independent contribution of CARand other risk factors including age, diabetes, smoking, neutrophil count, volume of radiocontrast media, eGFR and LVEF) to the development of CI-AKI. All confidence intervals were $95 \%$. P values $<0.05$ were considered statistically significant. All statistical analyses were performed by using the SPSS statistical software, version 23.0 (Statistical Package for the Social Sciences, - IBM, Chicago, IL, USA). Non-parametric variables were marked with '*' in all tables.

\section{Results}

Among the 210 patients enrolled in the study (mean age $65.8 \pm 12.8,29 \%$ were male), the incidence of CIAKI was $42 \%$ (n: 89). The patients with CI-AKI were had a higher frequency of diabetes mellitus, hypertension and family history of coronary artery disease than those without $(p<0.05$, for all). The baseline cholesterol and triglyceride (TG) levels were higher in patients with CI-AKI than those without. After onset of CI-AKI, the peak uric aside, white blood cell count (WBC), neutrophil count (NC), $s \mathrm{Cr}, \mathrm{CRP}$ and the increase in $\mathrm{s} \mathrm{Cr}$ levels were higher in patients with CI-AKI than those without, while

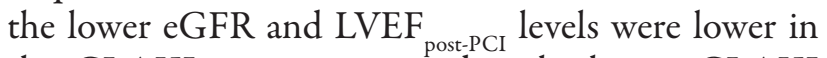
the CI-AKI group compared with the no-CI-AKI group $(\mathrm{p}<0.05$, for all). On the day when the $\mathrm{sCr}$ level peaked, the total fluid intake was higher in patients with CI-AKI than those without (2004.89 \pm 793.56 vs0.1857.35 \pm 558.57$)$, but the difference was not statistically significant $(\mathrm{p}=0.146)$. The total urine output was lower in patients with CI-AKI than those without (888.21 \pm 520.38 vs. $1066.83 \pm 556.09)$, and the difference between the groups was statistically significant $(\mathrm{p}=0.030)$. The CAR value $[1.159(0.502-2.970)$ vs $0.515(0.288-0.949) ; \mathrm{p}<0.001)]$ was higher in patients with CI-AKI than those without. Except for 1 stent implantation in the CI-AKI groups, no significant difference was found for 2 and 3 stent implantations in the CI-AKI groups and CAR tertiles. There was no significant difference in terms of infarct related artery (IRA) and stent diameter but, implanted stent length was longer in patients with CI-AKI than those without [median: 24 (interquartile ratio (IQR): 18-28.5 $\mathrm{mm}$ ) vs median: 22 (IQR: 18-26 mm), p=0.030]. Although, the in-hospital mortality rate was found to be higher in CI-AKI group (6.7\% vs $2.5 \%$ ), it was not 
statistically significant $(\mathrm{p}=0.132)$. Looking at the time when serum creatinine peaks, it was longer in patients with CI-AKI group than those without $(72.91 \pm 30.37$ vs. $62.44 \pm 25.16, \mathrm{p}<0.010)$. Similarly, the mean of the time of CAR was found to be longer in patients with CI-AKI group than those without $(56.2 \pm 26.73$ vs. 53.14 $\pm 25.04, \mathrm{p}<0.001)$. The mean of the overall time of CAR was found to be shorter than the mean of the overall time of peak $\mathrm{s} \mathrm{Cr}$ in all patients with $\mathrm{AMI}$ undergoing pPCI ( $54.43 \pm 25.74$ vs. $66.75 \pm 27.83$, $\mathrm{p}<0.001)$. The demographic, clinical, laboratory and angiographic characteristic of the patients were given in Table 1.

The CAR values of the patients were ranged from the smallest (0.014) to the largest (9.987), and divided into three groups with equal number of patients (70 patients in each group). Patients' CAR values $<0.415$ was included in to the lower CAR tertile, those with $>0.415$ to $\leq 1.076$ was included in to the mid-CAR tertile, and those with $>1.076$ was included in to the high-CAR tertile. The distribution of patients' characteristics into the three tertiles was given in Table 2. The rate of patients with CI-AKI was significantly higher in high CAR tertile than the mid- and low-CAR tertiles $(65.7 \%$ vs $31.4 \%$ vs $30 \%$, respectively; $\mathrm{p}<0.001)$. As in CI-AKI group, patients in the high-CAR tertiles had a higher rate of smoking, hypertension and diabetes mellitus compared to the patient in the midand low-CAR tertiles $(\mathrm{p}=0.028, \mathrm{p}=0.034, \mathrm{p}<0.001$, respectively).

Except from the basal lymphocyte count (LC), no significant difference was detected between the basal LC between the CAR tertiles $(p=0.442 p=0.394$ $\mathrm{p}=0.019)$. However, with the increasing $\mathrm{sCr}$ values, there was an increase in the peak NC while there was a decrease in the peak LC $(p<0.001, p<0.001, p=0.012$, respectively). Moreover, the development of CI-AKI was significantly increased as the median levels of CRP increased ( 0.909 vs 2.583 vs 7.552 , respectively; $\mathrm{p}<0.001$ ), while the median levels of lower ALB value decreased (3.74 vs 3.58 vs 3.21, respectively; $\mathrm{p}<0.001$ ) (Figure 1).

The in-hospital mortality rate was found to be higher in patients with high-CAR tertile (10\%) than those with low-CAR (1.4\%) and mid-CAR tertile (1.4\%) $(\mathrm{p}=0.015)$.

The correlation analyzes between peak CAR, CRP, sALB and demographic, clinical, laboratory and angiographic characteristic of the patients were given in Table 3. A significant correlation was found between the peak CRP and DM, peak sCr, increase of $s \mathrm{Cr}$, baseline sALB, lower sALB, decrease of sALB values, lower eGFR, decrase of eGFR, LVEF, CM volume and syntax score. Similarly, a significant correlation was found between the lower sALB values and gender, $\mathrm{DM}$, smoking, $\mathrm{TG}$, peak $\mathrm{s} \mathrm{Cr}$, increase of $\mathrm{s} \mathrm{Cr}$, baseline CRP, peak CRP, increase of CRP, lower eGFR, CM volume and syntax score. A significant correlation was also found between the CAR and DM, increase of sCr, lower eGFR, decreases of eGFR and LVEF values; but there was no significant correlation between CAR and gender, smoking, TG, baseline sCr, all eGFR values and AMI type. The correlation graphics between the CAR, increase of $\mathrm{s} C \mathrm{r}$ and decrease of $\mathrm{sALB}$ values were shown on Figure 2.

To determine the independent predictors of the development of CI-AKI, univariate and multivariate logistic regression analyses with an enter model were performed using the variables included gender, smoker, diabetes mellitus, dyslipidemia, hypertension, peak NC, peak CRP, lower sALB, CAR, LVEF ${ }_{\text {post-PCI }}$ and the CM volume. Except from the gender and dyslipidemia, other variables were showed the significant and marginal association with CI-AKI in the univariate logistic regression analyses $(p<0.05$, for all). Age, hypotension, hemoglobin and hematocrit values, which are the known risk factors for the CI-AKI, were not included in the regression analysis models because the study groups were matched age groups and anemia and hypotensive patients were not included in the study. Also, to prevent the multicollinearity statistic problem, CRP and albumin, which constitute the CRP to albumin ratio, were not included in the multivariate regression analysis module. In the multivariate regression analysis, smoking (OR: 2.366, 95\% CI: 1.117-5.010; $\mathrm{p}=0.024$ ), DM (OR: 3.949, 95\% CI: 1.792-8.701; $\mathrm{p}=0.001$ ), HT (OR: 2.647, 95\% CI: 1.257-5.573; $\mathrm{p}=0.010$ ), CAR (OR: 1.345, 95\% CI: 1.009-1.794; $\mathrm{p}=0.043$ ), LVEF (OR: 0.924, 95\% CI: 0.871-0.980; $\mathrm{p}=0.008$ ), peak NC (OR: 1.162, 95\% CI: $1.028-$ 1.313; $\mathrm{p}=0.016$ ) and the CM volume (OR: 1.007 , 95\% CI: $1.001-1.012 ; \mathrm{p}=0.012)$ were found to be an independent predictors of CI-AKI (Table 4).

Receiver operating characteristics (ROC) curve analysis was performed to determine whether CAR was a strength predictor than CRP and albumin in predicting CI-AKI. The area under the ROC curve (AUC) 
Table 1. The demographic, clinical, laboratory (baseline and peak value of $s C r$, and sCRP and lower value of $s A L B$ within 24 to 96 hours following the intravascular administration of $\mathrm{CM}$ ), echocardiographic and coronary angiographic characteristics of all patients, patients with without the no-Cl-AKI and Cl-AKI.

\begin{tabular}{|c|c|c|c|c|c|c|c|c|}
\hline \multirow[b]{2}{*}{ Variable } & & \multicolumn{6}{|c|}{ Contrast induced acute kidney injury (Cl-AKI) } & \multirow{3}{*}{$\begin{array}{c}\mathrm{p} \\
0.055\end{array}$} \\
\hline & & \multicolumn{2}{|r|}{$\begin{array}{l}\text { Overall } \\
n=210\end{array}$} & \multicolumn{2}{|c|}{$\begin{array}{c}\text { No-Cl-AKI } \\
n=121\end{array}$} & \multicolumn{2}{|c|}{$\begin{array}{l}\mathrm{Cl}-\mathrm{AKI} \\
\mathrm{n}=89\end{array}$} & \\
\hline \multicolumn{2}{|l|}{ Age (years) } & 65.8 & \pm 12.8 & 64.3 & \pm 12.03 & 67.8 & \pm 13.5 & \\
\hline \multicolumn{2}{|c|}{ Male gender, n (\%) } & 149 & (71) & 84 & (69.4) & 65 & (73) & 0.340 \\
\hline \multicolumn{2}{|l|}{ BMI $\left(\mathrm{kg} / \mathrm{m}^{2}\right)$} & 26.32 & \pm 4.74 & 25.89 & \pm 4.77 & 26.92 & \pm 4.65 & 0.117 \\
\hline \multicolumn{2}{|c|}{ STEMI, n (\%) } & 165 & (78.6) & 84 & (69.4) & 81 & (91) & $<0.001$ \\
\hline \multicolumn{2}{|c|}{ Hypertension, n (\%) } & 92 & (43.8) & 42 & $(34.7)$ & 50 & (56.2) & 0.002 \\
\hline \multicolumn{2}{|c|}{ Diabetes mellitus, $\mathrm{n}(\%)$} & 74 & $(35.2)$ & 22 & (18.2) & 52 & (58.4) & $<0.001$ \\
\hline \multicolumn{2}{|c|}{ Dyslipidemia, n (\%) } & 75 & $(35.7)$ & 49 & (40.5) & 26 & (42.4) & 0.092 \\
\hline \multicolumn{2}{|c|}{ Smoking, n (\%) } & 87 & (41.4) & 41 & (33.9) & 46 & (51.7) & 0.010 \\
\hline \multicolumn{2}{|c|}{ Family history, n (\%) } & 66 & (31.7) & 35 & (28.9) & 31 & (35.6) & 0.305 \\
\hline \multicolumn{2}{|c|}{$\mathrm{SBP}(\mathrm{mmHg})$} & 129.5 & (115.8-149.3) & 127 & $(114-142.5)$ & 137 & (118-162.5) & $0.018^{*}$ \\
\hline \multicolumn{2}{|c|}{$\mathrm{DBP}(\mathrm{mmHg})$} & 83 & \pm 13.2 & 81.4 & \pm 12.3 & 84.1 & \pm 14.1 & 0.148 \\
\hline Hemoglobin & & 14.04 & \pm 2.15 & 13.98 & \pm 2.14 & 14.12 & \pm 2.19 & 0.776 \\
\hline $\mathrm{BGL}(\mathrm{mg} / \mathrm{dl})$ & & 131 & $(108.75-163)$ & 124 & (104-154) & 143 & $(117-168)$ & $0.015^{*}$ \\
\hline Baseline Ch & (mg/dl) & 165.3 & \pm 36.9 & 170.8 & \pm 35.4 & 157.98 & \pm 37.7 & 0.019 \\
\hline Baseline LD & & 98.7 & \pm 34.17 & 100.96 & \pm 33.8 & 95.8 & \pm 34.7 & 0.302 \\
\hline Baseline HD & & 38 & (31.95-45.3) & 38 & (31.8-44.3) & 39.5 & $(31.9-5)$ & $0.702^{*}$ \\
\hline Baseline TG & & 111 & (87.8-150.8) & 125 & $(93.8-172)$ & 98.5 & $(78.8-129)$ & $0.001^{*}$ \\
\hline Peak uric ac & & 5.35 & $(4.5-6.3)$ & 5.1 & $(4.2-6.1)$ & 5.6 & $(4.8-6.9)$ & $0.036^{*}$ \\
\hline Baseline NC & & 5.3 & $(4.18-7.53)$ & 5.1 & $(4.1-6.4)$ & 5.5 & $(4.2-9.2)$ & $0.111^{*}$ \\
\hline Peak NC (10 & & 7.52 & $(5.7-10.1)$ & 6.7 & (5.51-9.3) & 9.7 & $(6.38-11.56)$ & $<0.001^{*}$ \\
\hline Baseline LC & & 1.6 & $(1.24-2.22)$ & 1.7 & $(1.3-2.3)$ & 1.55 & $(1.1-2.1)$ & $0.041^{*}$ \\
\hline Lower LC (1 & & 1.6 & $(1.2-2.2)$ & 1.7 & $(1.21-2.3)$ & 1.47 & $(1.095-2)$ & $0.242^{*}$ \\
\hline Fluid intake & & 1919.99 & \pm 670.43 & 1857.35 & \pm 558.57 & 2004.89 & \pm 793.56 & 0.146 \\
\hline Urine output & & 990.99 & \pm 546.93 & 1066.83 & \pm 556.09 & 888.21 & \pm 520.38 & 0.030 \\
\hline Baseline eGI & & 92.8 & (76.5-102.5) & 92.8 & (79.9-101.4) & 92.98 & 72.2-104.4) & $0.974^{*}$ \\
\hline Lower eGFR & & 74.5 & (55.13-87.02 & 78.3 & $(65.05-93.16)$ & 62.37 & (47.41-80.94) & $<0.001^{*}$ \\
\hline Decrease in & $1 / \min )$ & 13.67 & (7.98-24.18) & 11.18 & $(5.13-15.93)$ & 24.73 & (12.52-30.46) & $<0.001^{*}$ \\
\hline Decrease in & & 17.6 & (9.4-27.5) & 13.09 & $(6.5-19.4)$ & 30.6 & $(17.8-36.9)$ & $<0.001^{*}$ \\
\hline Baseline $\mathrm{sCr}$ & & 0.80 & $(0.68-0.948)$ & 0.815 & $(0.713-0.968)$ & 0.775 & $(0.653-0.91)$ & 0.063 \\
\hline Peak sCr (m & & 1.035 & (0.883-1.208) & 1 & $(0.843-1.13)$ & 1.15 & $(1-1.430)$ & $<0.001$ \\
\hline Increase in s & & .21 & $(0.15-0.34)$ & 0.16 & $(0.1-0.2)$ & 0.37 & $(0.3-0.52)$ & $<0.001$ \\
\hline Increase in $\mathrm{s}$ & & 27.03 & (17.38-44) & 19.51 & (13.53-25) & 45.59 & $(38.4-58.48)$ & $<0.001$ \\
\hline Baseline CR & & 0.376 & $(0.198-0.829)$ & 0.351 & $(0.200-0.603)$ & 0.399 & $(0.168-1.171)$ & $0.083^{*}$ \\
\hline Peak CRP (n & & 2.538 & (1.160-4.998) & 1.917 & (1.050-3.422) & 3.958 & $(1.697-9.317)$ & $<0.001^{*}$ \\
\hline Baseline sAL & & 4.123 & (3.803-4.32) & 4.155 & $(3.85-4.358)$ & 4.070 & $(3.750-4.300)$ & $0.138^{*}$ \\
\hline Lower sALB & & 3.510 & \pm 0.460 & 3.619 & \pm 0.429 & 3.369 & \pm 0.465 & $<0.001$ \\
\hline CAR & & 0.705 & $(0.321-1.505)$ & 0.515 & $(0.288-0.949)$ & 1.159 & $(0.502-2.970)$ & $<0.001^{*}$ \\
\hline Time of peal & & 66.75 & $\pm 27.83 \quad p<0.001$ & 62.44 & \pm 25.16 & 72.91 & \pm 30.37 & 0.010 \\
\hline Time of CAR & & 54.43 & \pm 25.74 & 53.14 & \pm 25.04 & 56.2 & \pm 26.73 & 0.416 \\
\hline Peak tropon & & 52.9 & (17.68-180) & 38.39 & (14.03-106) & 79.6 & (25-180) & $0.058^{*}$ \\
\hline LMCA (critic & $n(\%)$ & 5 & (2.4) & 3 & (2.5) & 2 & (2.4) & 0.913 \\
\hline IRA, n (\%) & LAD & 85 & (40.5) & 52 & (43) & 33 & (37.1) & 0.390 \\
\hline & $\mathrm{RCA}$ & 72 & (34.3) & 36 & (29.8) & 36 & (40.4) & 0.107 \\
\hline & $C x$ & 37 & (17.6) & 23 & (19) & 14 & (15.7) & 0.586 \\
\hline & Other & 16 & (7.6) & 10 & (8.3) & 6 & (7.6) & 0.681 \\
\hline Stent, n (\%) & One & 182 & $\mathrm{p}=0.068$ & 110 & (90.9) & 72 & (80.9) & 0.035 \\
\hline & Two & 23 & (11) & 10 & (8.3) & 13 & (14.6) & 0.146 \\
\hline & Three & 5 & (2.4) & 1 & $(0.8)$ & 4 & (4.5) & 0.085 \\
\hline Stent diamet & & 3.03 & \pm 0.48 & 3.08 & \pm 0.499 & 2.98 & \pm 0.444 & 0.131 \\
\hline Stent length & & 22.5 & (18-27) & 22 & (18-26) & 24 & (18-28.5) & $0.030^{*}$ \\
\hline Radiocontras & (ml) & 198 & (150-250) & 200 & (150-200) & 200 & (200-300) & $<0.001$ \\
\hline Length hosp & (days) & 5 & $(4-6)$ & 4 & $(4-5)$ & 6 & $(4-7)$ & $<0.001$ \\
\hline $\mathrm{LVEF}_{\text {post-PCl}}(\%$ & & 50 & (46-54) & 54 & (50-55) & 47 & (40-49) & $<0.001$ \\
\hline Mortality, n ( & & 9 & (4.3) & 3 & $(2.5)$ & 6 & $(6.7)$ & 0.132 \\
\hline
\end{tabular}


Table 2. The demographic, clinical, laboratory, echocardiographic and coronary angiographic characteristics of patients with the low-CAR, mid-CAR and high-CAR.

\begin{tabular}{|c|c|c|c|c|c|c|c|c|}
\hline \multirow{3}{*}{ Age (years) } & & \multicolumn{7}{|c|}{ Peak C-reactive protein to lower albumin ratio (CAR) } \\
\hline & & \multicolumn{2}{|c|}{$\begin{array}{c}\text { Low-CAR }(\leq .415) \\
n=70\end{array}$} & \multicolumn{2}{|c|}{$\begin{array}{c}\text { Mid-CAR ( }>415 \text { to } \leq 1.076) \\
n=70\end{array}$} & \multicolumn{2}{|c|}{$\begin{array}{c}\text { High-CAR (>1.076) } \\
n=70\end{array}$} & \multirow{2}{*}{$\begin{array}{c}p \\
<0.001\end{array}$} \\
\hline & & 62.5 & \pm 10.7 & 63.3 & \pm 12.6 & 71.5 & \pm 12.99 & \\
\hline \multicolumn{2}{|c|}{ Male gender, $\mathrm{n}(\%)$} & 51 & (72.9) & 53 & $(75.7)$ & 45 & $(64.3)$ & 0.301 \\
\hline \multicolumn{2}{|l|}{$\mathrm{BMI}\left(\mathrm{kg} / \mathrm{m}^{2}\right)$} & 26.94 & \pm 4.5 & 26.5 & \pm 4.37 & 25.5 & \pm 5.25 & 0.205 \\
\hline \multicolumn{2}{|c|}{ Smoker, n (\%) } & 25 & $(35.7)$ & 24 & $(34.3)$ & 38 & $(41.4)$ & 0.028 \\
\hline \multicolumn{2}{|c|}{ Hypertension, n (\%) } & 24 & $(34.3)$ & 29 & (41.4) & 39 & $(55.7)$ & 0.034 \\
\hline \multicolumn{2}{|c|}{ Diabetes mellitus, $n(\%)$} & 12 & $(17.1)$ & 24 & $(34.3)$ & 38 & $(54.3)$ & $<0.001$ \\
\hline \multicolumn{2}{|c|}{ Family history, n (\%) } & 18 & (26.1) & 24 & (34.3) & 24 & $(34.8)$ & 0.467 \\
\hline \multicolumn{2}{|c|}{ Dyslipidemia, n (\%) } & 29 & (41.4) & 25 & $(35.7)$ & 21 & (30) & 0.370 \\
\hline \multicolumn{2}{|c|}{ Hemoglobin (mg/dl) } & 14.21 & \pm 2.12 & 14.22 & \pm 1.78 & 13.68 & \pm 2.53 & 0.544 \\
\hline \multicolumn{2}{|l|}{ BGL (mg/dl) } & 123 & $(103-150)$ & 131 & $(109-163)$ & 139 & (117.1-196.5) & $0.006^{*}$ \\
\hline \multicolumn{2}{|c|}{ Cholesterol (mg/dl) } & 165.6 & \pm 35.2 & 167.1 & \pm 36.8 & 163.3 & \pm 39.1 & 0.849 \\
\hline \multicolumn{2}{|l|}{ LDL (mg/dl) } & 94.9 & \pm 31.6 & 105.4 & \pm 34.6 & 96.1 & \pm 35.8 & 0.180 \\
\hline $\mathrm{HDL}$ (mg/dl) & & 40 & $(32.3-49)$ & 38 & $(31-44)$ & 38 & $(32-45)$ & $0.520^{*}$ \\
\hline Triglyceride & & 122.5 & $(88.3-171.3)$ & 123 & $(92.5-171)$ & 107 & $(82-138)$ & $0.259^{*}$ \\
\hline Baseline LC & & 5.4 & $(4.13-6.68)$ & 4.8 & $(4.23-7.07)$ & 5.8 & $(3.97-9.23)$ & $0.394^{*}$ \\
\hline Peak NC (10 & & 6.6 & $(5.4-9.3)$ & 7.2 & (5.195-9.75) & 8.569 & (6.3-11.73) & $<0.001^{*}$ \\
\hline Baseline NC & & 1.74 & $(1.34-2.3)$ & 1.7 & $(1.2-2.3)$ & 1.51 & $(1-2.01)$ & $0.019^{*}$ \\
\hline Peak LC (10 & & 1.84 & $(1.38-2.3)$ & 1.62 & $(1.2-2.55)$ & 1.41 & $(1.01-1.975)$ & $0.012^{*}$ \\
\hline Baseline sC & & .815 & $(0.698-0.965)$ & .805 & $(0.7-0.97)$ & 0.790 & $(0.67-0.91)$ & $0.675^{*}$ \\
\hline Peak sCr (m & & 1.03 & $(0.868-1.193)$ & 1.02 & $(0.878-1.153)$ & 1.115 & $(0.913-1.423)$ & 0.098 \\
\hline Increase of & & 0.2 & $(0.118-0.27)$ & 0.2 & $(0.13-0.33)$ & 0.31 & $(1.925-0.555)$ & $<0.001^{*}$ \\
\hline Increase of & & 18.8 & $(12.8-26.1)$ & 20.1 & $(14.8-26.6)$ & 30.2 & $(20-37.3)$ & $<0.001^{\circ}$ \\
\hline Baseline CR & & 0.221 & $(0.11-0.356)$ & 0.49 & $(0.279-0.839)$ & 0.674 & $(0.277-2.283)$ & $<0.001^{*}$ \\
\hline Peak CRP $(n$ & & 0.909 & $(0.614-1.195)$ & 2.583 & (1.931-3.189) & 7.552 & (5.015-11.933) & $<0.001^{*}$ \\
\hline Increase in & & 0.6 & $(0.287-0.998)$ & 1.938 & (1.396-2.513) & 6.878 & $(4.231-10.769)$ & $<0.001^{*}$ \\
\hline Increase in & & 73.2 & $(53.09-84.1)$ & 78.7 & $(65.8-85.8)$ & 90.8 & $(73.2-96.5)$ & $<0.001^{\circ}$ \\
\hline Baseline sA & & 4.23 & $(4.085-4.393)$ & 4.15 & (3.815-4.365) & 3.95 & (3.703-4.165) & $<0.001^{*}$ \\
\hline Lower sALB & & 3.74 & \pm 0.37 & 3.58 & \pm 0.38 & 3.21 & \pm 0.46 & $<0.001$ \\
\hline Decrease of & & 0.39 & $(0.208-0.678)$ & 0.49 & $(0.298-0.7)$ & 0.595 & $(0.34-1.013)$ & $0.003^{*}$ \\
\hline Decrease of & & 9.8 & $(5.1-14.7)$ & 12.2 & $(7.3-16.4)$ & 16 & $(9.01-24.1)$ & $<0.001^{*}$ \\
\hline Baseline eG & & 93.7 & $(79.2-104.1)$ & 93.2 & $(75.5-103.6)$ & 90 & (75.4-98.2) & $0.250^{\star}$ \\
\hline Lower eGFR & & 76.2 & $(63.7-88.4)$ & 76.8 & $(57.9-90)$ & 66.6 & $(45.6-81.9)$ & $0.007^{*}$ \\
\hline Decrease of & (min) & 12.8 & $(5.99-21.1)$ & 13.8 & $(8.3-19.7)$ & 19.6 & $(8.8-28.3)$ & $0.027^{*}$ \\
\hline Decrease of & & 14.9 & $(6.97-25.2)$ & 17.3 & $(9.1-25)$ & 23 & $(11.2-33.9)$ & $0.002^{*}$ \\
\hline CAR & & 0.254 & $(0.162-0.327)$ & .712 & $(0.533-0.872)$ & 2.420 & (1.517-4.032) & $<0.001^{*}$ \\
\hline Cl-AKI, n (\% & & 21 & (30) & 22 & (31.4) & 46 & $(65.7)$ & $<0.001$ \\
\hline STEMI, n (\% & & 54 & (77.1) & 51 & (72.9) & 60 & $(85.7)$ & 0.168 \\
\hline Peak tropon & & 40.28 & $(3.45-172.75)$ & 55.75 & (15.33-180) & 58.67 & (25-180) & 0.347 \\
\hline LMCA (critic & $n(\%)$ & 1 & (1.4) & 2 & (2.9) & 2 & (2.4) & 0.815 \\
\hline IRA, n (\%) & LAD & 22 & (31.4) & 32 & (45.7) & 31 & (44.3) & 0.166 \\
\hline & $C x$ & 15 & (21.4) & 10 & (14.3) & 12 & (17.1) & 0.536 \\
\hline & $\mathrm{RCA}$ & 24 & (34.3) & 23 & (32.9) & 25 & (35.7) & 0.939 \\
\hline & Other & 9 & (12.9) & 5 & (7.1) & 2 & (2.9) & 0.082 \\
\hline Stent, $\mathrm{n}(\%)$ & One & 64 & (91.4) & 61 & $(87.1)$ & 57 & (81.4) & 0.218 \\
\hline & Two & 5 & (7.1) & 8 & (11.4) & 10 & (14.3) & 0.395 \\
\hline & Three & 1 & (1.4) & 1 & (1.4) & 3 & $(4.3)$ & 0.441 \\
\hline Stent diame & & 3.03 & \pm 0.54 & 2.99 & \pm 0.43 & 3.07 & \pm 0.46 & 0.568 \\
\hline Stent length & & 23 & $18-26$ & 22 & $18-26$ & 24 & $19-28.3$ & $0.146^{*}$ \\
\hline Radiocontra & & 200 & 73.2 & 189.7 & 81.5 & 212.2 & 80.3 & 0.436 \\
\hline Length of $\mathrm{hc}$ & , (days) & 4 & $(4-5)$ & 5 & $(4-5)$ & 6 & $(5-7)$ & $<0.001^{*}$ \\
\hline LVEF (\%) & & 53 & (49-55) & 51.5 & $(47.75-54)$ & 48 & $(40-50)$ & $<0.001^{*}$ \\
\hline Syntax scor & & 18.95 & (13.9-22.3) & 19 & (13.8-26) & 25.8 & $(16-32.93)$ & $0.002^{*}$ \\
\hline Mortality, $\mathrm{n}$ & & 1 & (1.4) & 1 & (1.4) & 7 & (10) & 0.015 \\
\hline
\end{tabular}




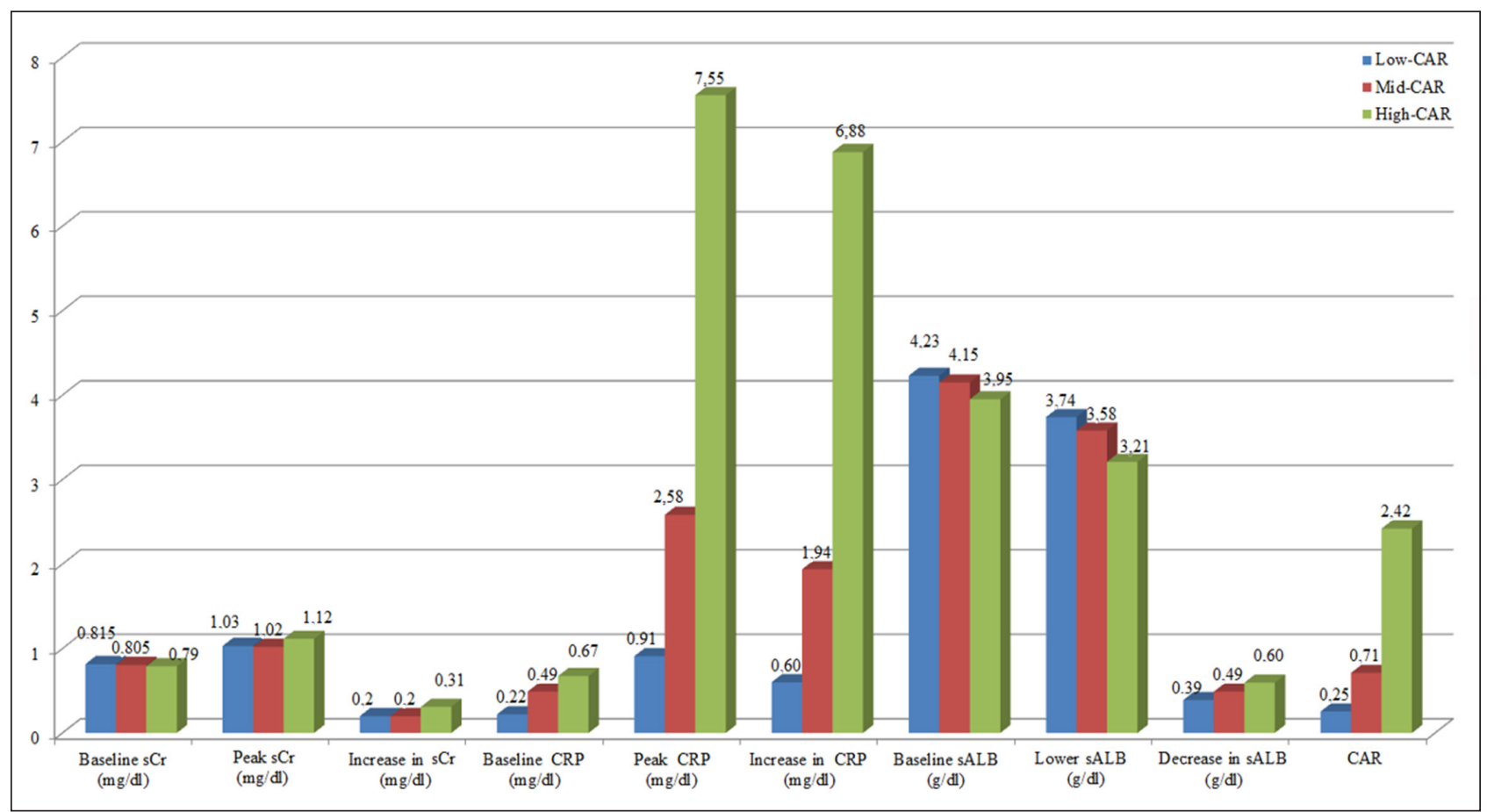

Figure 1. The median concentrations of serum creatinine (sCr), serum albumin (SALB), C-reactive protein (CRP), and C-reactive protein to lower albumin rate (CAR) with the baseline, peak, and amount of increase in all patients before and after primary percutaneous intervention divided into the low-CAR (blue), mid-CAR (red) and high-CAR (green) groups.

Table 3. Correlation analysis between demographic, clinical, biochemical, echocardiographic and coronary angiographic characteristics of all patients and with the correlation coefficient $r$ and $p$ value.

\begin{tabular}{|c|c|c|c|c|c|c|c|c|}
\hline & \multicolumn{2}{|c|}{ Increases in $\mathrm{sCr}$} & \multicolumn{2}{|c|}{ Peak CRP } & \multicolumn{2}{|c|}{ Lower sALB } & \multicolumn{2}{|c|}{ CAR } \\
\hline & $r$ & $p$ & $r$ & $\mathrm{p}$ & $r$ & $\mathrm{p}$ & $r$ & $\mathrm{p}$ \\
\hline Gender & 0.044 & 0.530 & -0.076 & 0.275 & 0.202 & 0.003 & -0.084 & 0.225 \\
\hline Diabetes mellitus & 0.358 & $<0.001$ & 0.249 & $<0.001$ & -0.331 & $<0.001$ & 0.265 & $<0.001$ \\
\hline Smoker & 0.274 & $<0.001$ & 0.021 & 0.769 & -0.212 & 0.002 & 0.054 & 0.435 \\
\hline Triglyceride & -0.132 & 0.071 & 0.189 & 0.006 & 0.306 & $<0.001$ & -0.052 & 0.473 \\
\hline Baseline sCr & - & - & 0.018 & 0.791 & -0.038 & 0.580 & 0.026 & 0.704 \\
\hline Peak sCr & - & - & 0.171 & 0.013 & -0.219 & 0.001 & 0.183 & 0.008 \\
\hline Increases in sCr & - & - & 0.255 & $<0.001$ & -0.313 & $<0.001$ & 0.268 & $<0.001$ \\
\hline Peak NC & 215 & 0.002 & 0.336 & $<0.001$ & -0.227 & 0.001 & 360 & $<0.001$ \\
\hline Baseline CRP & 0.199 & 0.004 & - & - & -0.271 & $<0.001$ & - & - \\
\hline Peak CRP & 0.255 & $<0.001$ & - & - & -0.375 & $<0.001$ & - & - \\
\hline Increase in CRP & 0.237 & 0.001 & - & - & -0.349 & $<0.001$ & - & - \\
\hline Baseline SALB & -0.243 & $<0.001$ & -0.167 & 0.016 & - & - & - & - \\
\hline Lower SALB & 0.313 & $<0.001$ & -0.375 & $<0.001$ & - & - & - & - \\
\hline Decrease in SALB & 0.104 & 0.135 & 0.261 & $<0.001$ & - & - & - & - \\
\hline CAR & 0.267 & $<0.001$ & - & - & - & - & - & - \\
\hline Baseline eGFR & - & - & -0.123 & 0.076 & 0.184 & 0.007 & -0.128 & 0.064 \\
\hline Lower eGFR & - & - & -0.227 & 0.001 & 0.267 & $<0.001$ & -0.231 & 0.001 \\
\hline Decrease in GFR & - & - & 0.169 & 0.015 & -0.134 & 0.052 & 0.250 & $<0.001$ \\
\hline AMI type (STEMI) & 0.143 & 0.039 & 0.059 & 0.398 & -0.140 & 0.043 & 0.046 & 0.511 \\
\hline LVEF $_{\text {post-PCI }}$ & -0.387 & $<0.001$ & 0.403 & $<0.001$ & -0.037 & 0.597 & -0.405 & $<0.001$ \\
\hline CM volume & 0.398 & $<0.001$ & -0.140 & 0.043 & 0.243 & $<0.001$ & 0.060 & 0.390 \\
\hline Syntax score & 0.207 & 0.003 & 0.201 & 0.004 & -0.323 & $<0.001$ & -0.155 & 0.025 \\
\hline
\end{tabular}



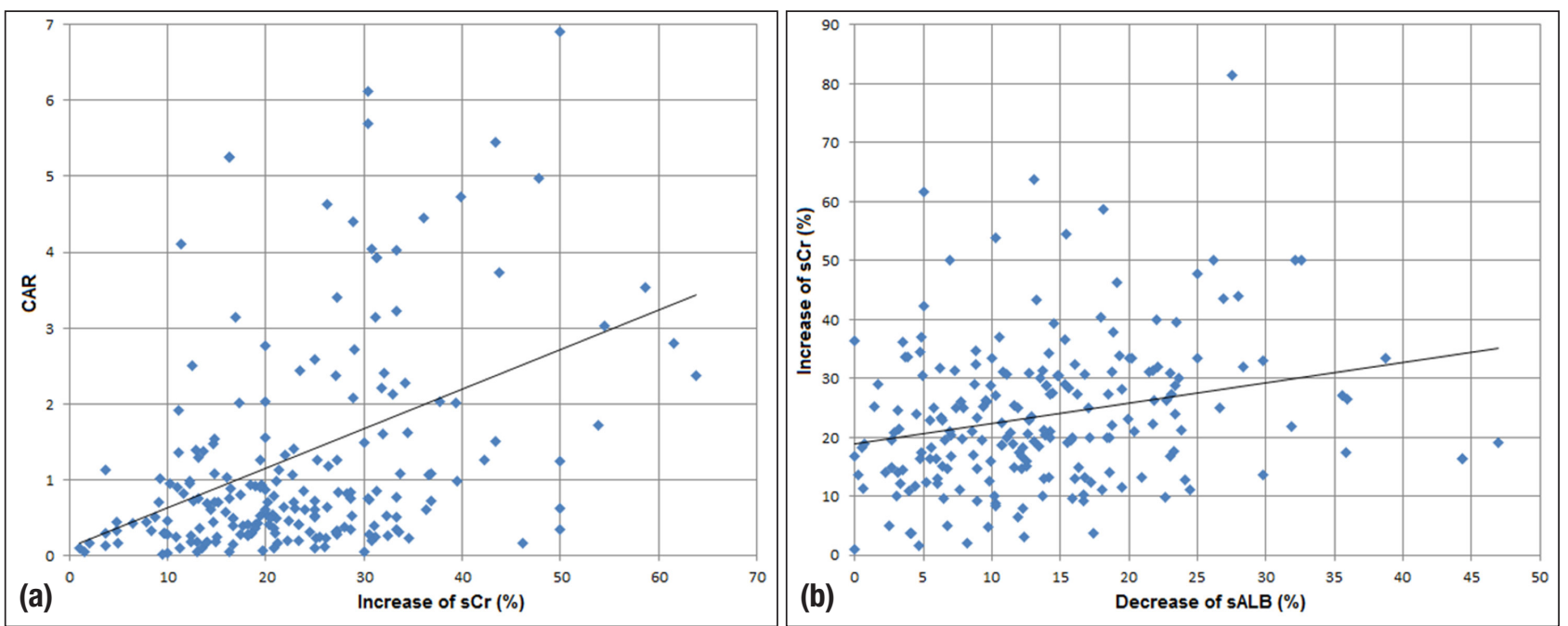

Figure 2. a, b. Correlation analysis between CAR and increase in $s C r$ values [correlation coefficient $(r)=0.357 ; p<0.001$ (a)] and between increase in sCr and decrease in $S A L B$ values $[r=0.248 ; p<0.001$ (b)]

Table 4. The logistic regression analysis models of CAR for prediction of contrast-induced acute kidney injury

\begin{tabular}{|c|c|c|c|c|c|}
\hline & $\beta$ & S.E. & $\mathrm{OR}$ & 95\% C.I. & $\mathrm{p}$ values \\
\hline \multicolumn{6}{|c|}{ Univariate regression analysis } \\
\hline Gender & 0.176 & 0.310 & 1.193 & $0.650-2.189$ & 0.569 \\
\hline Smoker & 0.736 & 0.286 & 2.087 & $1.191-3.657$ & 0.010 \\
\hline Diabetes mellitus & 1.844 & 0.319 & 6.324 & $3.384-11.820$ & $<0.001$ \\
\hline BGL & 0.003 & 0.002 & 1.003 & $.999-1.007$ & 0.114 \\
\hline Dyslipidemia & -0.500 & 0.298 & 0.606 & $0.338-1.087$ & 0.093 \\
\hline Hypertension & 0.880 & 0.287 & 2.411 & $1.375-4.229$ & 0.002 \\
\hline Peak NC & 0.188 & 0.049 & 1.207 & $1.097-1.329$ & $<0.001$ \\
\hline Peak CRP & 0.176 & 0.041 & 1.193 & $1.101-1.292$ & $<0.001$ \\
\hline Lower SALB & -1.279 & 0.342 & 0.278 & $0.142-0.545$ & $<0.001$ \\
\hline CAR & 0.495 & 0.120 & 1.640 & $1.296-2.076$ & $<0.001$ \\
\hline LVEF $_{\text {post-PCI }}$ & -0.136 & 0.026 & 0.873 & $0.830-0.918$ & $<0.001$ \\
\hline CM volume & 0.008 & 0.002 & 1.008 & $1.004-1.012$ & $<0.001$ \\
\hline \multicolumn{6}{|c|}{ Multivariate regression analysis } \\
\hline Smoker & 0.861 & 0.383 & 2.366 & $1.117-5.010$ & 0.024 \\
\hline Diabetes mellitus & 1.374 & 0.403 & 3.949 & $1.792-8.701$ & 0.001 \\
\hline Hypertension & 0.973 & 0.380 & 2.647 & $1.257-5.573$ & 0.010 \\
\hline Peak NC & 0.150 & 0.062 & 1.162 & $1.028-1.313$ & 0.016 \\
\hline CAR & 0.297 & 0.147 & 1.345 & $1.009-1.794$ & 0.043 \\
\hline LVEF $_{\text {post-PCI }}$ & -0.079 & 0.030 & 0.924 & $0.871-0.980$ & 0.008 \\
\hline CM volume & 0.007 & 0.003 & 1.007 & $1.001-1.012$ & 0.012 \\
\hline
\end{tabular}

$\mathrm{Cl}$, confident interval; OR, odds ratio; SE, standard error.

of CAR (AUC 0.689, 95\% CI 0.614-0.763; p<0.001) was significantly higher than that of CRP (AUC: 0.679 , 95\% CI $0.604-0.754 ; \mathrm{p}<.001)$ and $\mathrm{sALB}$ (AUC: $0.322,95 \%$ CI $0.249-0.395 ; \mathrm{p}<0.001$ ). It was demonstrated that CAR was found to be the strongest, CRP was moderate strength, and SALB was a poor predictor for CI-AKI (Figure 3).

\section{Discussion}

Present study demonstrated that, the CAR was associated with the occurrence of CI-AKI in patients with non-ST- and ST-elevation AMI, who had undergone pPCI, and also it was an independent predictor of CI-AKI development. Moreover, the CAR predicted 


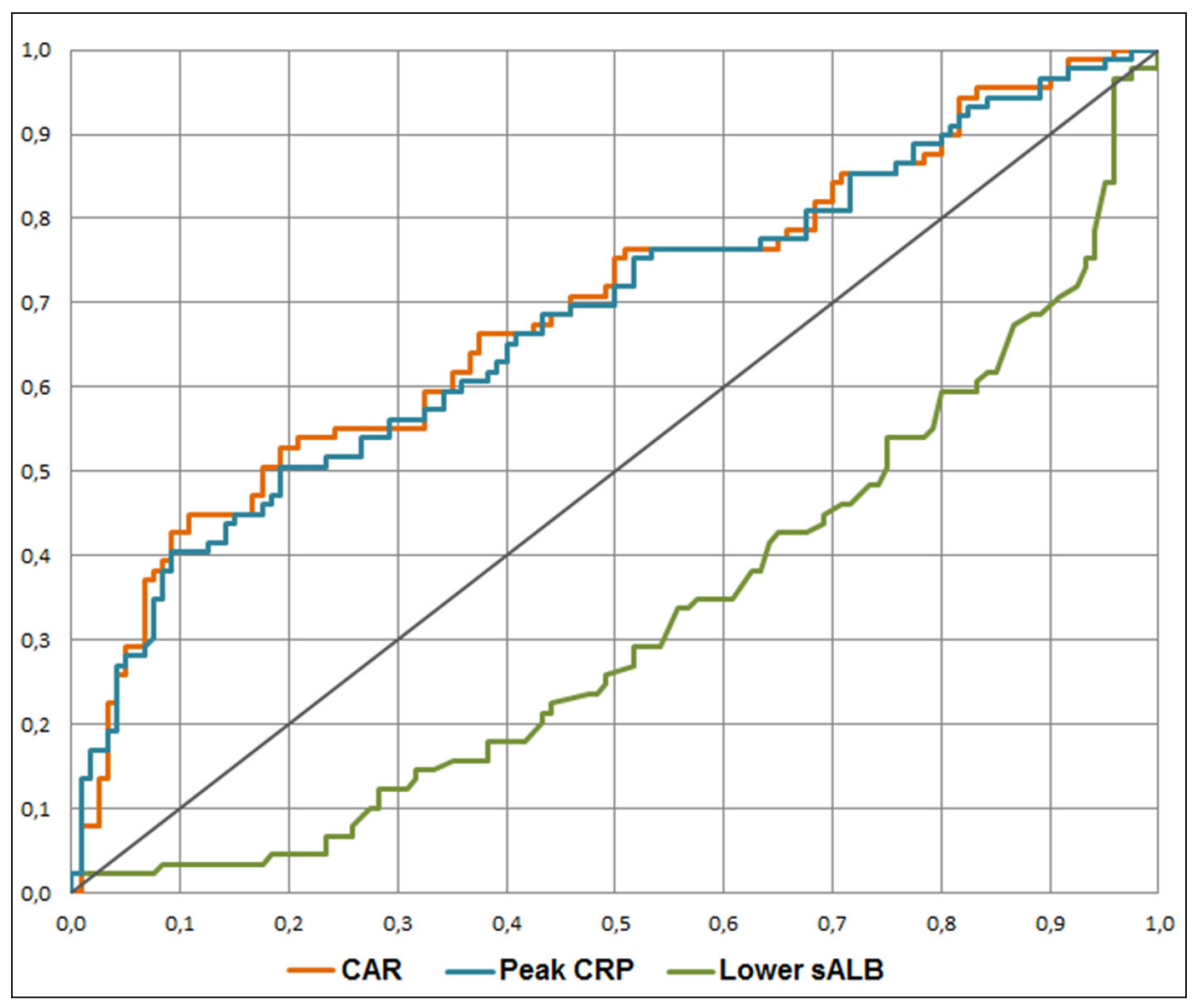

Figure 3. The receiver operator characteristic (ROC) curve analysis. The area under the ROC curve for predicting contrast-induced acute kidney injury of CAR, peak CRP, and lower SALB were 0.689 [95\% confidence interval (Cl): 0.614-0.763; $p<0.001], 0.679$ (95\% Cl: $0.604-0.754 ; p<0.001)$ and $0.322(95 \% \mathrm{Cl}$ : $0.249-0.395 ; p<0.001)$, respectively.

CI-AKI more accurately than either CRP or sALB. Furthermore, present study demonstrated that smoking, DM, HT, elevated CAR and elevated NC, using of high CM volume and lower LVEF are risk factors for CI-AKI.

All CM have more or less renal toxic effects. Their toxic effect is closely related to its osmolality, viscosity and iodine concentration. Although the use of hyperosmolar CM has been abandoned at present time, iso-osmolar and hypo-osmolar CM are also more viscous than plasma, which is inversely proportional to osmolality. The toxic effects of the CM have been shown to be directly related to its properties ${ }^{15}$. Although, the pathophysiologic mechanism of development CI-AKI is not clearly understood, the suggested pathophysiologic mechanisms of the development of CI-AKI are complex including induced and prolongation of intrarenal arterial vasoconstriction with resultant medullary hypoxia, renal vascular endothelial damage, generation of reactive oxygen species (ROS), and direct renal tubular toxicity ${ }^{16-18}$. In previous studies conducted in animal models have shown that intra-arterial infusion of increased dose of ionic-contrast media causes a transient increase of renal blood flow, and then subsequently causes intense and prolonged vasoconstriction related the volume of CM leading to renal ischemic injury/ infarct $^{19,20}$. This effect decreases blood flow of approximately $40 \%$ and oxygen supply of $60 \%$, especially in the outer medulla ${ }^{21-23}$. This effect has also been shown to be due to the local balance between endothelial vasoconstrictors such as endothelin ${ }^{19}$ and adenosine ${ }^{20}$ and vasodilators such as nitrous oxide ${ }^{24}$ and prostaglandin ${ }^{25}$ has been shown an impaired in favor of intrarenal arterial vasoconstrictors caused ischemic injury/infarct. In our study, it was shown that the CI-AKI group had higher the median of CM volume and increase of $s \mathrm{Cr}$. And also, it was shown that the increases of $\mathrm{sCr}$ values showed significant correlation with the CM volumes. These findings support the results observed in animal models, which the increased CM volume causes more renal ischemic injury/infarct ${ }^{21,22}$. The development chain of CI-AKI was shown on Figure 4.

\section{CRP and $\mathrm{Cl}-\mathrm{AKI}$}

CRP primarily made in the liver in response to proinflammatory cytokines, in particular interleukin-6 is the non-specific acute phase reactant, and is a widely used biochemical marker for acute inflammation ${ }^{26}$. 


\section{Contrast media (CM) administration}

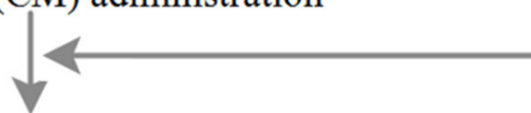

Endothelial damage

Prolongation of intrarenal arterial vasoconstriction

Release of vasoactive substance

(e.g. nitrous oxide, endothelin, adenosine)

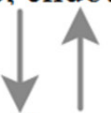

Inflammation/inflammatory response

(e.g. increases of CRP and CAR, decreases of sALB)

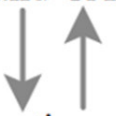

Generation of toxic reactive oxygen species (ROS)

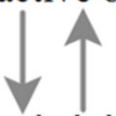

Renal ischemic injury/infarct

Deterioration of renal function parameters

(Decreases/absent of eGFR and urine output, increase in $\mathrm{sCr}$ )
Periprocedural CI-AKI risk factor $^{3}$ :

- Hypotension

- Intra-arterial balloon pump therapy

- Chronic heart failure, (NYHA

III/IV or recent pulmonary edema)

- Age $>75$ years

- Diabetes mellitus

- Anemia (male: $\mathrm{HCT}<0.39$, female: HCT<0.36)

- Estimated glomerular filtration rate $<60 \mathrm{~mL} / \mathrm{min}$

- $\mathrm{CM}$ volume

Pre-procedural risk factor ${ }^{3}$ :

- Prior CM exposure within $72 \mathrm{~h}$

- Left ventricular ejection fraction $<45 \%$

- Pre-procedure $\mathrm{sCr}>$ baseline $\mathrm{sCr}$

- Baseline $\mathrm{sCr}>1.5 \mathrm{mg} / \mathrm{dL}$

-Diabetes mellitus

- Creatinine clearance (eGFR) $<44$ $\mathrm{mL} / \mathrm{min}$

- Age $>73$ years

Figure 4. The development chain of the contrast-induced acute kidney injury (Cl-AKI).

The increased plasma concentration of CRP is indicative of systemic inflammation, and a few studies have shown that increased CRP concentration is association with acute contrast-induced renal injury and an independent predictor of CI-AKI development ${ }^{23-29}$. In present study, the median of CRP level was also significantly higher in the both CI-AKI group than the no-CI-AKI group and high-CAR tertile than the low-CAR and mid-CAR tertiles. In addition, a strong positive correlation was found between CRP and $s \mathrm{Cr}$ levels. Moreover, ROC analysis showed that CRP is an independent predictor in the development of CI-AKI. The results of our study are accordance with the results of previous studies. Although the relationship of CRP with CI-AKI is clearly not understood, the ability to promote precipitation of polycationic and polyanionic compounds, activation of the classical complement pathway, inhibition of endothelial nitric oxide synthase, impaired vascular reactivity, agglutination properties were thought a played role for development of CI-AKI.

\section{Albumin and $\mathrm{Cl}-\mathrm{AKI}$}

Serum albumin is a well-known negative acute phase reactant, which is a good indicator of inflammatory process. In the setting of systemic inflammation, it has been shown to reduce albumin levels regardless of the patient's nutritional status ${ }^{6}$ and to be an important predictor of poor prognosis in acute coronary syndromes ${ }^{30-32}$.

Murat et al. ${ }^{33}$ demonstrated that low sALB pre-PCI increases the risk of developing CI-AKI in patients with acute coronary syndrome. In the present study, the median of sALB level was significantly lover in the both CI-AKI group than the no- CI-AKI group and high-CAR tertile than the mid-CAR and low-CAR tertiles. In addition, a strong negative correlation was found between sALB and sCr level. Moreover, ROC analysis showed that $\mathrm{SALB}$ is an independent predictor in the development of CI-AKI. These findings suggest that albumin plays a prominent role in the pathophysiological mechanism of development of CI-AKI. In this 
study, the relationship between sALB and the development of CI-AKI was demonstrated for the first time, and was shown to be an independent predictor in patient with AMI undergoing pPCI.

Although the relationship between hypoalbuminemia and CI-AKI has been showed in patient with administered CM, its role in the mechanism of development of CI-AKI is unknown.

Albumin has been shown to act as a reservoir for short-lived free nitric oxide (NO). When $\mathrm{PH}$ falls due to tissue hypoxia, albumin-linked $\mathrm{NO}$ is released to maintain vascular ton $\mathrm{e}^{34}$. Hypoxia by prolonged vasoconstriction induced by CM due to decreased $\mathrm{NO}$ concentration in renal tissue due to hypoalbuminemia may be one of the mechanisms leading to CI-AKI development ${ }^{35}$. Approximately $60 \%$ of the whole body albumin mass is in the interstitial space and $40-45 \%$ is in the vascular space, and accounts for $75-80 \%$ of normal plasma oncotic pressure ${ }^{36,37}$. Hypoalbuminemia reduces blood oncotic pressure and induces escape of fluid from intracellular space towards the interstitial space. The expanded interstitial space causes a relative decrease in hypovolemia in the intravascular space. Thus, the toxic effect of CM may be increased because of the relative decreased intra vascular volume due to hypoalbuminemia and increase of their distribution space.

Finally, the present and previous studies have shown a strong inversely correlation between sALB level and the magnitude of inflammation. In addition, hypoalbuminemia can increase blood viscosity by reducing erythrocyte flexibility and increasing the level of fibrinogen and impair endothelial function. Moreover, ALB has antioxidant activity. In the sitting of acute high grate inflammation, the effect on endothelial function and damage, the increased blood viscosity and reduced antioxidant activity, hypalbuminemia may cause the development of CI-AKI by augmenting of the toxic effect of $\mathrm{CM}$.

\section{CAR and Cl-AKI}

The CAR, which is consists of CRP to SALB ratio, was first reported in patient with acute medical admissions to the acute medical assessment unit for patient outcome $^{38}$. And also, it has been shown that elevated CAR is associated with poor prognosis in cardiovascular diseases and a predictor for cardiovascular events ${ }^{3}$.

It's well known that elevated CRP and reduced sALB were inversely related in patient with inflammatory disease. This inverse relationship between CRP and
sALB levels in inflammatory disease and the predictive values of CAR derived from these biomarkers in the development of CI-AKI has led us to investigate in patient with non-ST- and ST-elevation AMI undergoing pPCI.

In our study, the CI-AKI group had significantly higher median of CAR than the no-CI-AKI group. Similarly, the high-CAR tertile had significantly higher the median of peak $s \mathrm{Cr}$ value, the absolute increase in $\mathrm{s} \mathrm{Cr}$ and the percentage increase in $\mathrm{s} \mathrm{Cr}$ than the midCAR and low-CAR. Although CAR groups were had small size, according to the ROC analyze, the AUC value of the CAR was found to be higher than CRP and sALB alone. Thus, our findings suggest that CAR has had more predictive value than CRP and sALB alone for predicting the development of CI-AKI in patients with AMI undergoing pPCI.

This study showed a significant association between CAR and SALB in the development and progression of CI-AKI and demonstrated that they are independent predictors of the development of the development of CI-AKI in patients with AMI undergoing pPCI. Given that CAR has a higher predictive value than CRP and sALB alone, it is a more appropriate tool to use for predicting the development of CIN in patients with AMI undergoing pPCI. In addition, patients with an elevated CAR may require more intensive therapy for the prevention or progression of CI-AKI.

Thus, we believe that our results point to a promising, simple, cheap and useful risk classification tool for predicting the development and progression of CI-AKI for all patients receiving $\mathrm{CM}$.

\section{Limitations}

Our study had some limitations. Firstly, it was a retrospective study and the study group consisted of patient enrolled in a single center. Secondly, in this study, which has a limited number of patients, the effect of CAR on cardiac events could not be evaluated. Thirdly, as we exclude high-risk patients such as used IABP or mechanical ventilation, hemodialysis and a history of coronary artery bypass grafting, the findings may not be used for this group of patients. However, we believe that CAR and ALB have the importance of being used as predictor of post-processing CI-AKI in most patients undergoing pPCI. Finally, this study reflects the predictive value of CAR for the period of hospitalization in predicting CIAKI. Therefore, large-scale and long-term studies are needed to determine the long-term predictive capacity. 


\section{Financial Disclosure}

The author received no specific funding for this work.

\section{Conflicts of Interests}

The authors report no conflicts of interest.

\section{References}

1. Nash K, Hafeez A, Hou S. Hospital-acquired renal insufficiency. Am J Kidney Dis 2002;39(5):930-6.

2. Gupta R, Birnbaum Y, Uretsky BF. The renal patient with coronary artery disease: current concepts and dilemmas. J Am Coll Cardiol 2004;44(7):1343-53.

3. Rear R, Bell RM, Hausenloy DJ. Contrast-induced nephropathy following angiography and cardiac interventions. Heart 2016;102(8):638-48.

4. Kwasa EA, Vinayak S, Armstrong R. The role of inflammation in contrast-induced nephropathy. The British journal of radiology 2014;87(1041):20130738-

5. Herrmann J, Lennon RJ, Barsness GW, Sandhu GS, Gulati R, Best PJ et al. High sensitivity C-reactive protein and outcomes following percutaneous coronary intervention in contemporary practice. Circ Cardiovasc Interv 2012;5(6):783-90.

6. Ishida S, Hashimoto I, Seike T, Abe Y, Nakaya Y, Nakanishi H. Serum albumin levels correlate with inflammation rather than nutrition supply in burns patients: a retrospective study. J Med Invest 2014;61(3-4):361-8.

7. Soeters PB, Wolfe RR, Shenkin A. Hypoalbuminemia: Pathogenesis and Clinical Significance. JPEN J Parenter Enteral Nutr 2019;43(2):181-93.

8. Wu J, Tan W, Chen L, Huang Z, Mai S. Clinicopathologic and prognostic significance of C-reactive protein/albumin ratio in patients with solid tumors: an updated systemic review and meta-analysis. Oncotarget 2018;9(17):13934-47.

9. Kim MH, Ahn JY, Song JE, Choi H, Ann HW, Kim JK et al. The C-Reactive Protein/Albumin Ratio as an Independent Predictor of Mortality in Patients with Severe Sepsis or Septic Shock Treated with Early Goal-Directed Therapy. PLoS One 2015;10(7): e0132109.

10. Ibanez B, James S, Agewall S, Antunes MJ, Bucciarelli-Ducci C, Bueno H et al 2017 ESC Guidelines for the management of acute myocardial infarction in patients presenting with STsegment elevation: The Task Force for the management of acute myocardial infarction in patients presenting with ST-segment elevation of the European Society of Cardiology (ESC). Eur Heart J 2018;39(2):119-77.

11. Bellomo R, Ronco C, Kellum JA, Mehta RL, Palevsky P. Acute renal failure - definition, outcome measures, animal models, fluid therapy and information technology needs: the Second International Consensus Conference of the Acute Dialysis Quality Initiative (ADQI) Group. Crit Care 2004;8(4): R204-12.
12. Mehta RL, Kellum JA, Shah SV, Molitoris BA, Ronco C, Warnock DG et al. Acute Kidney Injury Network: report of an initiative to improve outcomes in acute kidney injury. Crit Care 2007;11(2): R31.

13. Morcos SK, Thomsen HS, Webb JA. Contrast-media-induced nephrotoxicity: a consensus report. Contrast Media Safety Committee, European Society of Urogenital Radiology (ESUR). Eur Radiol 1999;9(8):1602-13.

14. eGFR using CKD-EPI. Calculate by QxMD, https://qxmd. $\mathrm{com} /$ calculate/calculator_251/egfr-using-ckd-epi [accessed 30 06 19]

15. Pannu N, Wiebe N, Tonelli M. Prophylaxis strategies for contrast-induced nephropathy. Jama 2006;295(23):2765-79.

16. Goldenberg I, Matetzky S. Nephropathy induced by contrast media: pathogenesis, risk factors and preventive strategies. Cmaj 2005;172(11):1461-71.

17. McCullough PA. Acute kidney injury with iodinated contrast. Crit Care Med 2008;36(4 Suppl): S204-11.

18. McCullough PA, Adam A, Becker CR, Davidson C, Lameire N, Stacul F et al. Risk prediction of contrast-induced nephropathy. Am J Cardiol 2006;98(6a):27k-36k.

19. Heyman SN, Clark BA, Kaiser N, Spokes K, Rosen S, Brezis M et al. Radiocontrast agents induce endothelin release in vivo and in vitro. J Am Soc Nephrol 1992;3(1):58-65.

20. Klause N, Arendt T, Lins M, Gronow G. Hypoxic renal tissue damage by endothelin-mediated arterial vasoconstriction during radioangiography in man. Adv Exp Med Biol 1998;454:225-34.

21. Bakris GL, Burnett JC, Jr. A role for calcium in radiocontrastinduced reductions in renal hemodynamics. Kidney Int 1985;27(2):465-8.

22. Liss P, Nygren A, Olsson U, Ulfendahl HR, Erikson U. Effects of contrast media and mannitol on renal medullary blood flow and red cell aggregation in the rat kidney. Kidney Int 1996;49(5):1268-75.

23. Nygren A. Contrast media and regional renal blood flow. A study of the effects of ionic and non-ionic monomeric and dimeric contrast media in the rat. Acta Radiol Suppl 1992;378(Pt 3):123-35.

24. Schwartz D, Blum M, Peer G, Wollman Y, Maree A, Serban I et al. Role of nitric oxide (EDRF) in radiocontrast acute renal failure in rats. Am J Physiol 1994;267(3 Pt 2): F374-9.

25. Heyman SN, Reichman J, Brezis M. Pathophysiology of radiocontrast nephropathy: a role for medullary hypoxia. Invest Radiol 1999;34(11):685-91.

26. Hurlimann J, Thorbecke GJ, Hochwald GM. The liver as the site of C-reactive protein formation. J Exp Med 1966;123(2):365-78.

27. Liu Y, Tan N, Zhou YL, Chen YY, Chen JY, Chen J et al. High-sensitivity C-reactive protein predicts contrast-induced nephropathy after primary percutaneous coronary intervention. J Nephrol 2012;25(3):332-40.

28. Jian-Wei Z, Yu-Jie Z, Shu-Jun C, Qing Y, Shi-Wei Y, Bin N. Impact of preprocedural high-sensitivity C-reactive protein on contrastinduced nephropathy in patients undergoing primary percutaneous coronary intervention. Angiology 2014;65(5):402-7. 
29. Lazaros G, Zografos T, Oikonomou E, Siasos G, Georgiopoulos G, Vavuranakis M et al. Usefulness of C-Reactive Protein as a Predictor of Contrast-Induced Nephropathy After Percutaneous Coronary Interventions in Patients With Acute Myocardial Infarction and Presentation of a New Risk Score (Athens CIN Score). Am J Cardiol 2016;118(9):1329-33.

30. Schalk BW, Visser M, Bremmer MA, Penninx BW, Bouter LM, Deeg DJ. Change of serum albumin and risk of cardiovascular disease and all-cause mortality: Longitudinal Aging Study Amsterdam. Am J Epidemiol 2006;164(10):969-77.

31. Oduncu V, Erkol A, Karabay CY, Kurt M, Akgun T, Bulut M et al. The prognostic value of serum albumin levels on admission in patients with acute ST-segment elevation myocardial infarction undergoing a primary percutaneous coronary intervention. Coron Artery Dis 2013;24(2):88-94.

32. Kurtul A, Ocek AH, Murat SN, Yarlioglues M, Demircelik $\mathrm{MB}$, Duran $\mathrm{M}$ et al. Serum albumin levels on admission are associated with angiographic no-reflow after primary percutaneous coronary intervention in patients with ST-segment elevation myocardial infarction. Angiology 2015;66(3):278-85.
33. Murat SN, Kurtul A, Yarlioglues M. Impact of Serum Albumin Levels on Contrast-Induced Acute Kidney Injury in Patients With Acute Coronary Syndromes Treated With Percutaneous Coronary Intervention. Angiology 2015;66(8):732-7.

34. Minamiyama Y, Takemura S, Inoue M. Albumin is an important vascular tonus regulator as a reservoir of nitric oxide. Biochem Biophys Res Commun 1996;225(1):112-5.

35. Vuong TD, Braam B, Willekes-Koolschijn N, Boer P, Koomans HA, Joles JA. Hypoalbuminaemia enhances the renal vasoconstrictor effect of lysophosphatidylcholine. Nephrol Dial Transplant 2003;18(8):1485-92.

36. Fleck A, Raines G, Hawker F, Trotter J, Wallace PI, Ledingham IM et al. Increased vascular permeability: a major cause of hypoalbuminaemia in disease and injury. Lancet 1985;1(8432):781-4.

37. Don BR, Kaysen G. Serum albumin: relationship to inflammation and nutrition. Semin Dial 2004;17(6):432-7.

38. Fairclough E, Cairns E, Hamilton J, Kelly C. Evaluation of a modified early warning system for acute medical admissions and comparison with C-reactive protein/albumin ratio as a predictor of patient outcome. Clin Med (Lond) 2009;9(1):30-3. 